\title{
Reuna
}

\section{EFEITOS DA INOVAÇÃO ORGANIZACIONAL NO DESEMPENHO DA ATENÇÃO INTEGRAL EM DOENÇA DE CHAGAS}

\author{
EFFECTS OF ORGANIZATIONAL INNOVATION IN THE INTEGRAL HEALTHCARE IN \\ CHAGAS DISEASE
}

http://dx.doi.org/10.21714/2179-8834/2016v21n4p57-80

\author{
Marcelino José Jorge \\ Fundação Oswaldo Cruz, Instituto Nacional de Infectologia Evandro Chagas, Brasil. \\ Endereço: Avenida Brasil, 4365 - INI, Manguinhos, CEP 21045900 - Rio de Janeiro, RJ - Brasil \\ Fone: +55 (21) 38659542 \\ Email: marcelino.jorge@ipec.fiocruz.br - Lattes: http://lattes.cnpq.br/6837125666966113 \\ Alejandro Marcel Hasslocher Moreno \\ Fundação Oswaldo Cruz, Instituto Nacional de Infectologia Evandro Chagas, Brasil. \\ Endereço: Avenida Brasil, 4365 - INI, Manguinhos, CEP 21045900 - Rio de Janeiro, RJ - Brasil \\ Fone: +55 (21) 38659542 \\ Email: alejandro.hasslocher@ini.fiocruz.br - Lattes: http://lattes.cnpq.br/3808469339011845
}

\section{Andréa Silvestre de Sousa}

Fundação Oswaldo Cruz, Instituto Nacional de Infectologia Evandro Chagas, Brasil.

Endereço: Avenida Brasil, 4365 - INI, Manguinhos, CEP 21045900 - Rio de Janeiro, RJ - Brasil Fone: +55 (21) 38659542

Email: andrea.silvestre@ini.fiocruz.br - Lattes: http://lattes.cnpq.br/0990926729685349

\section{Patrícia Santos Cavalheiro Silva}

Fundação Oswaldo Cruz, Instituto Nacional de Infectologia Evandro Chagas, Brasil.

Endereço: Avenida Brasil, 4365 - INI, Manguinhos, CEP 21045900 - Rio de Janeiro, RJ - Brasil Fone: +55 (21) 38659542

Email: patricia.cavalheiro@ini.fiocruz.br - Lattes: http://lattes.cnpq.br/4127796251579092

\section{Georg Michael Lennart Weinberg}

Fundação Oswaldo Cruz, Instituto Nacional de Infectologia Evandro Chagas, Brasil.

Endereço: Avenida Brasil, 4365 - INI, Manguinhos, CEP 21045900 - Rio de Janeiro, RJ - Brasil Fone: +55 (21) 38659542

Email: georgweinberg@gmail.com - Lattes: http://lattes.cnpq.br/0753593687385379

Submissão: 21 Out. 2016 Publicação: 30 Dez. 2016. Sistema de avaliação: Double blind review. Centro Universitário UNA, Belo Horizonte - MG, Brasil. Editor geral Prof. Dr. Mário Teixeira Reis Neto

Este artigo encontra-se disponível no endereço eletrônico:

Reuna: http://revistas.una.br/index.php/reuna/article/view/823

DOI - http://dx.doi.org/10.21714/2179-8834/2016v21n4p57-80

\section{RESUMO}

Evidências sobre a eficiência do Modelo de Atenção Integral em saúde no uso de recursos podem promover a sua difusão. O Laboratório de Pesquisa Clínica em Doença de Chagas do Instituto Nacional de Infectologia Evandro Chagas da 
Fundação Oswaldo Cruz adotou a estrutura Organização Inovadora para desenvolver a pesquisa clínica associada à assistência. O objetivo do artigo é avaliar esse exemplo de inovação organizacional para agregar conhecimento sobre o princípio direcionador da inovação na organização pública multipropósito de saúde e contribuição gerencial sobre a descentralização orientada para a inovação. Usa os métodos de levantamento de microcustos e Custeio Baseado em Atividades, o teste de correlação de Spearman e o Modelo DEA na avaliação. Confirma, quanto ao princípio direcionador da inovação, a contribuição para: o desenvolvimento da pesquisa; a diversificação e a efetividade em custos na distribuição dos recursos da assistência; e a eficiência relativa do conjunto destas atividades.

Palavras-chave: Inovação Organizacional; Atenção Integral em Saúde; Desempenho em Custos; Eficiência.

\begin{abstract}
Evidence on efficient use of resources can promote the diffusion of the Integral Healthcare Model. The Laboratory for Clinical Research in Chagas Disease of the Evandro Chagas National Institute of Infectious Diseases, Oswaldo Cruz Foundation (Lapclin-Chagas) adopted the Innovative Organization structure in 2007 with view to develop clinical research about Chagas disease. Using the methods of surveying of microcosts and Activity Based Costing, the Spearman correlation test and the DEA model, the paper aims to assess this organizational innovation in order to aggregate knowledge about the driving principle of innovation in the multipurpose public health organization and bring a managerial contribution on innovation-oriented decentralization. It shows its effect to develop research, diversify as well as promote cost-effectiveness and overall efficiency gains in the distribution of resources to healthcare activities. It aggregates knowledge about the effects coming from innovation-oriented decentralization and from the diffusion of the experience of the Lapclin-Chagas.
\end{abstract}

Keywords: Organizational Innovation; Integral Healthcare; Cost Effectiveness; Efficiency.

\title{
Introdução
}

Segundo a literatura sobre vantagens competitivas, as organizações internalizam a orientação empreendedora para alcançar suas metas com mais eficiência, bem como conviver com a concorrência e adaptar-se à mudança; a estrutura da organização deve ser menos centralizada e envolver mais participação, e as organizações públicas responsáveis pelas soluções mais inovadoras desfrutam de estruturas descentralizadas. A propósito da estrutura das organizações de saúde, essa literatura descarta o risco de duplicação das atividades gerenciais e de queda da produtividade associada à descentralização da governabilidade da atenção.

Na Fundação Oswaldo Cruz (Fiocruz), por exemplo, as limitações atribuídas à sua organização, segundo o modelo Organização Profissional e o modelo Organização Divisionada, resultaram na escolha da configuração Organização Inovadora (MINTZBERG, 1995) em 2006 e da subestrutura Laboratório de Pesquisa Clínica Associada à Assistência para a reestruturação do Instituto Nacional de Infectologia 
Evandro Chagas da Fiocruz (INI) em 2007, com vistas a melhorar o seu desempenho na pesquisa inovadora, mediante a incorporação de características de autonomia e de orientação empreendedora entre seus profissionais.

A valorização dos princípios da "nova gestão pública", em busca de sustentabilidade do posicionamento do INI na pesquisa clínica sobre doenças infecciosas, a preocupação quanto aos recursos necessários ao tratamento em saúde, segundo os princípios do Modelo de Atenção Integral e a promoção da inovação, resultaram na estratégia de aliar esforços de inovação organizacional pró-orientação empreendedora na atenção clínica em doenças infecciosas à busca de eficiência no uso de recursos e de ganhos de efetividade clínica nos esquemas de tratamento.

Nesse sentido, o INI adotara a estrutura organizacional Ação Integrada (PAI) em 1999, associando atividades de assistência, ensino e pesquisa. A PAl sobre doença de Chagas do Laboratório de Pesquisa Clínica em Doença de Chagas (LapclinChagas) foi uma experiência de integração dessas atividades consolidada a partir da reestruturação do INI em 2007.

Este artigo avalia a evolução recente da pesquisa clínica em doença de Chagas no INI, com vistas a agregar conhecimento sobre o efeito da descentralização da estrutura organizacional na promoção da pesquisa e na melhoria do desempenho nas atividades de assistência organizadas segundo os princípios do Modelo de Atenção Integral em saúde. A questão focalizada neste artigo diz respeito à investigação dos tipos e efeitos das soluções organizacionais - ou mecanismos internos de incentivo - que potencializam os resultados da interação entre as atividades de pesquisa e de assistência (FIOCRUZ, 2006) através da motivação continuada para a pesquisa inovadora. A formulação do problema de avaliação do desempenho do INI resultou da hipótese de que a solução dos problemas internos de compromisso, através do foco na pesquisa orientada por missões, promove o crescimento da pesquisa clínica.

O método de pesquisa escolhido foi o estudo de caso único sobre a eficácia do modelo (BANA e COSTA, 2000) do Lapclin-Chagas, uma fonte de evidências pouco explorada, com vistas a agregar conhecimento empírico acerca do efeito da descentralização das formas de alocação e coordenação da força de trabalho (MINTZBERG, 1995) sobre o desempenho.

Analisando os dados disponíveis a respeito da evolução recente das despesas anuais e dos custos unitários efetivos dos laboratórios do INI, busca evidenciar os efeitos positivos das novas subestruturas resultantes da mudança organizacional de 2007 sobre a evolução da distribuição dos recursos destinados às atividades de atenção clínica do INI entre os seus laboratórios, bem como do grau de diversificação da assistência e do desempenho em custos da atenção clínica prestada no Lapclin-Chagas no período 2006-2011.

Em seguida, recorre ao resultado da análise do desempenho conjunto das diversas atividades das PAI do INI para avaliar a eficácia dessa estrutura de atenção integral, que incorpora atividades de ensino e pesquisa, utilizando um modelo de representação adequada das especificidades do funcionamento do INI como unidade produtiva e do seu processo de tomada de decisão sobre a alocação de recursos no período 2002-2012. 
O artigo focaliza a quantificação, aparentemente inédita na literatura, do grau de diversificação dos procedimentos e da evolução do desempenho, em termos de efetividade em custo e eficiência, do Modelo de Atenção Integral no tratamento da doença de Chagas.

\section{Marco conceitual}

Os conceitos necessários à avaliação da inovação na organização pública multipropósito de saúde - aqui conceituada como uma organização pública complexa, sujeita a conflitos internos de interesses, uma unidade produtiva prestadora de serviços de atenção integral em saúde associados ao ensino e à pesquisa que utiliza recursos especializados, muitos comercializados em mercados incompletos, para produzir "bens públicos", e cuja avaliação está sujeita à experiência de uso, assim como contribuir para o desenvolvimento da prestação de serviços em saúde - foram estabelecidos: na literatura de Ciência da Saúde sobre a mudança institucional orientada para o Modelo de Atenção Integral em saúde; na literatura de Administração e Economia sobre as relações entre a centralização da tomada de decisão, a vantagem competitiva da firma e o posicionamento estratégico da organização pública; e na literatura de Análise de Eficiência sobre a tomada de decisão na organização produtiva com informação incompleta.

\subsection{Atenção integral e diversificação na organização pública multipropósito de saúde}

A literatura discute os fundamentos do Modelo de Atenção Integral que podem influenciar a sua adoção na organização pública multipropósito de saúde.

O modelo é associado à importância das ações de promoção da saúde, de prevenção das doenças e de recuperação, vis-à-vis o enfoque curativo centrado na doença, e aos princípios jurídico-legais das práticas de saúde orientadas pela busca de precedência dos direitos humanos sobre as relações de mercado, norteadores da constituição do Sistema Único de Saúde brasileiro (SUS).

É caracterizado como modelo de transição entre a assistência médica ambulatorial centrada no trabalho médico especializado e a atenção centrada no trabalho multiprofissional; como instrumento de transformação, de reinserção social do paciente e de promoção da intersetorialidade das ações, de redução de danos e de avanço nas políticas públicas que repercutem na prática cotidiana das unidades de saúde.

Finalmente, é destacada a garantia de maior acesso e gratuidade dos serviços que o modelo confere à população, diante: das mudanças na autonomia dos pacientes; das novas tecnologias médicas; e da inexistência de mecanismos formais para resolver os problemas eticamente complexos resultantes (ULLOA GONZALEZ; BARRANTES MONGE, 2008). Com respeito a essa garantia, é prescrita a passagem de um modelo de atenção centrada no hospital para um modelo de maior envolvimento familiar e social no processo de reabilitação, que dê conta da complexidade das relações entre o profissional de saúde e os pacientes.

REUNA, Belo Horizonte - MG, Brasil, v.21, n.4, p.57-80, Dut. - Dez. 20I6 - ISSN 2179-8834 
Marcelina Jasé Jarge, Alejandra Marcel Hasslacher Marena, Andréa Silvestre de Sausa, Patrícia Santas Cavalheiro Silva, Gearg Michael Lennart Weinberg

A literatura sobre o chamado Integral Healthcare Model destaca que o modelo aperfeiçoa a prescrição de procedimentos e de instruções de interação do profissional de saúde com o paciente (ROSS, 2009). Sustenta ainda que, face à focalização nos aspectos biológicos, as percepções desses profissionais e dos acompanhantes domésticos devem diferir e que esta abordagem deve ser substituída por uma lógica "multiconceitual" e multidisciplinar (OLIVEIRA; NASCIMENTO; MARCOLINO, 2012).

Esclarece, a propósito, que os serviços considerados no seu contexto abrangem até mesmo o suprimento de água limpa para o usuário e o ensino de hábitos saudáveis. Para o profissional de saúde, esses serviços contemplam a inclusão do paciente e da comunidade, o auxílio dos pares e o acesso aos recursos necessários. Além disso, requerem o controle de um amplo leque de recursos, tais como hospitais, instalações complementares e de distribuição de atenção, médicos, enfermeiros, técnicos especializados, engenheiros e sanitaristas.

Finalmente, a opção pelo Modelo de Prestação de Serviços de Saúde Abrangentes, designação alternativa do modelo, orientada para a proteção social, não só considera o efeito esperado para a prevenção diante dos riscos de perda de saúde, mas em termos de garantia de serviços de atenção de qualidade, de prevenção quanto a dificuldades financeiras provenientes de despesas de saúde elevadas e da eficácia dos programas de saúde pública dirigidos a diferentes coortes de pacientes, através do controle do comportamento de risco (KNAUL et al., 2012; IVERS et al., 2011).

\subsection{Descentralização e inovação na organização pública multipropósito de saúde}

$\mathrm{Na}$ origem do desenvolvimento dos laboratórios do INI, a organização dos Institutos da Fiocruz como Organização Profissional (MINTZBERG, 1995), constituída por profissionais com alta padronização das habilidades, em que o trabalho é complexo e prevalece a contratação de profissionais treinados e detentores do controle sobre o seu próprio trabalho, com vistas à internalização destes padrões, enfatizava a autoridade de natureza profissional, mas caracterizava-se por baixa governabilidade dos objetivos organizacionais.

Quanto à Organização Divisionada, configuração que sucedeu o modelo Organização Profissional, suas unidades operacionais são diferenciadas pela padronização dos outputs, têm autonomia e pouca hierarquia, mas encontram-se submetidas ao controle uniforme de um "escritório central" e o parâmetro-chave do desenho da organização é o sistema de controle de desempenho (MINTZBERG, 1995). Essa configuração, no entanto, adotada com o propósito de promover a pesquisa "estratégica" sobre saúde através do modelo de gestão orientada para resultado, assegurou autonomia restrita aos Institutos da Fiocruz a partir de 1995, com outputs escolhidos a priori, através da definição do plano estratégico com seu "escritório central" apoiada na avaliação de desempenho.

Nesse contexto, o INI integrou as atividades de diagnóstico laboratorial, atenção clínica, ensino e pesquisa das doenças infecciosas focalizadas pelo seu plano estratégico em estruturas organizacionais de tipo PAl em 1999, com o objetivo de 
tornar-se referência e mobilizar recursos para a pesquisa clínica (JORGE et al., 2006).

Em sintonia com o paradigma da "nova gestão pública", a busca de posicionamento estratégico do INI resultou, ainda, na preocupação de aliar ganhos de eficiência no uso de recursos aos ganhos de efetividade clínica obtidos nos esquemas de tratamento adotados, mediante a operação de mecanismos de incentivo, como a orientação para resultados, a descentralização dos controles gerenciais, a responsabilização e a flexibilização de procedimentos (CUNILL GRAU; OSPINA BOZZI, 2003).

De fato, a geração de conhecimento sobre doenças infecciosas foi definida como produto-padrão do INI, mas a restrição à autonomia da pesquisa pela padronização das atividades também comprometeu a promoção da pesquisa clínica.

Em 2007, então, a reestruturação dos Institutos pelo modelo Organização Inovadora focalizou o incentivo à orientação inovadora com empoderamento do núcleo operacional, descentralização, liberdade de "ajuste mútuo" entre profissionais para a constituição das equipes de pesquisa e condicionamento da criação e sobrevivência dos laboratórios pelos seus resultados (MINTZBERG, 1995).

Em consequência, foram criados os Laboratórios de Pesquisa Clínica do INI, subestruturas concebidas para assumir vínculos de resposta rápida aos usuários das pesquisas, com vistas a promover a pesquisa inovadora (FIOCRUZ, 2006). Entre os laboratórios, o Lapclin-Chagas exemplifica uma experiência consolidada de atenção integral.

Nesse sentido, a necessidade de internalizar a iniciativa e desenvolver o potencial do indivíduo resultou na importância conferida à escolha da estrutura organizacional detentora das condições para motivar a orientação empreendedora (MORTON; HU, 2008).

Combinadas, as características de autonomia e atitude competitiva, inovadora, próativa e de predisposição ao risco resultariam em melhorar o desempenho da organização, que lograria alcançar metas com mais eficiência e adquirir capacidade adaptativa a ambientes turbulentos (JOGARATNAM; CHING-YICK, 2006). Entre elas, a autonomia individual é considerada a influência direta de maior peso na orientação empreendedora. Uma conclusão da literatura é que a estrutura organizacional deve ser menos centralizada e envolver mais participação (ROBINSON, 2007).

Por seu turno, a atividade de pesquisa é sujeita a incertezas difíceis de avaliar, realizada em ambiente dinâmico e complexo, face ao que a integração das atividades da pesquisa clínica associada à assistência depende da interação efetiva, por ajuste mútuo, entre profissionais com diferentes padrões de habilidades (HARRIS, 1977) em equipes de projetos ad hoc e encoraja o desenvolvimento da Organização Inovadora.

$\mathrm{Na}$ Organização Inovadora, a delicada conciliação dos objetivos do núcleo operacional e da administração (HARRIS, 1977) não depende da coordenação externa por sistemas de regulação; a resposta à mudança externa pelo núcleo operacional reduz a necessidade de supervisão direta dos operadores pelos gerentes de linha, e o ajuste mútuo dos profissionais é o principal mecanismo de coordenação.

REUNA, Belo Horizonte - MG, Brasil, v.21, п.4, p.57-80, Dut. - Dez. 20I6 - ISSN 2179-8834 
Em consequência, a mudança organizacional da pesquisa clínica associada à assistência da Fiocruz em 2006 focalizou mecanismos de divisão de trabalho e coordenação da pesquisa que evoluam continuamente, de acordo com os projetos existentes, visando integrar as ações assistenciais estruturadas entre as modalidades (internação, hospital-dia e ambulatório), as linhas de cuidado (v.g. a infectologia), as atividades de apoio (v.g. a patologia clínica) e o suporte teórico (v.g. a epidemiologia clínica) aos laboratórios (FIOCRUZ, 2006).

\subsection{Informação, eficiência e avaliação na organização pública multipropósito de saúde}

Sob a ótica da Análise Econômica, a produção é organizada com o objetivo de obter eficiência no uso dos recursos, de forma que o estudo da busca de eficiência visa conhecer a unidade produtiva através da compreensão dos efeitos dos modelos gerenciais, das estratégias e das estruturas sobre sua criação e expansão (MILGROM; ROBERTS, 1992). Em outras palavras, a busca de eficiência explica a organização e expressa sua racionalidade, de modo que a prescrição da Análise Econômica para o funcionamento da unidade produtiva como organização econômica, no ambiente da competição, é buscar eficiência no uso dos recursos (VARIAN, 2006). A doutrina da chamada Administração Pública Gerencial, por exemplo, é considerada consistente com os princípios e políticas da Análise Econômica (LA FORGIA; COUTTOLENC, 2008).

Em particular, em presença de informação completa sobre o processo produtivo, a função objetivo de maximização do lucro da teoria neoclássica do equilíbrio da firma no curto prazo explica a busca de eficiência absoluta no uso dos recursos, enquanto a inovação "poupadora de custos" resulta do princípio direcionador da inovação na teoria neoclássica sobre a mudança técnica, a saber, a minimização de custos.

Com vistas à avaliação organizacional, então, a informação sobre custos pode aprimorar o desempenho das organizações (ATKINSON et al., 2000) e, no setor público, o controle dos custos pode contribuir para atender mais "clientes" (HORNGREN; SUNDEM; STRATTON, 2004).

Nesse sentido, além das ferramentas de controle, é necessário escolher o sistema de apropriação de custos, porque o processo de apropriação define a precisão dos resultados do custo do produto apurado, a qual motiva decisões gerenciais, tais como o incentivo ou não a uma linha de produto ou serviço (WARREN; REEVE; FESS, 2008).

Essa ótica, porém, costuma pressupor que existe um padrão de referência, a chamada "função custo", que retrata o custo de uma organização modelar, idealizada, devotada à minimização de custos (e/ou à maximização do lucro), que disporia a priori de informação completa sobre a melhor tecnologia e os preços de todos os fatores de produção recomendados pelas combinações técnicas possíveis. A convicção é de que a organização, assim como a firma estilizada que maximiza lucro, alcança o objetivo de sobrevivência no mercado através da busca de máxima eficiência. A economia nos custos e a inovação poupadora de custos expressam o sucesso do seu desempenho. 
No entanto, a hipótese de informação completa, incorporada aos modelos neoclássicos explicativos dos preços praticados e do tamanho da firma em concorrência perfeita, deixou de ser de interesse unânime na Análise Econômica.

Se, ao contrário, o gerente não conhece a priori, por completo, a tecnologia que a organização utiliza ou pode utilizar, nem os preços relativos dos fatores, nem o tamanho do mercado ou, ainda, se não pode observar o talento e o esforço dos subordinados, então não existe associação entre a avaliação acerca da eficiência relativa do gerente e a relação do custo efetivo observado da organização avaliada com o custo da organização benchmark.

Como alternativa, a moderna Economia Interna da Organização incorpora a hipótese de assimetria da informação (SOUZA; MAUSS; DIEHL, 2008) à descrição da interação entre o gerente e o agente executor da produção no interior da organização, do que resultam o interesse e a preferência pela formulação, dentre os modelos explicativos da organização através da eficiência de seu desempenho, daqueles que adotam a hipótese informacional de existência de ineficiências fora do controle do gerente, as chamadas ineficiências de tipo X (LEIBENSTEIN, 1966).

Como destaca a Teoria do Crescimento Endógeno, os conceitos de "conhecimento como bem livre", "mudança técnica exógena", "eficiência absoluta", "retornos de escala constantes" e "ausência de desequilíbrios com ineficiência no curto prazo" são concepções equivalentes à hipótese de informação completa do modelo de equilíbrio da firma em concorrência perfeita no curto prazo. Em contraste, o reconhecimento de ineficiências, do custo da informação e da informação incompleta, da heterogeneidade tecnológica, do aprendizado e dos retornos variáveis de escala distingue a axiomática dos modelos da firma em concorrência imperfeita (ROMER, 1994).

Quanto à natureza da inovação nas organizações, se disruptiva ou incremental, o "Modelo da Curva de Aprendizado" sustenta que a acumulação de conhecimento na organização inovadora é incremental no curto prazo (ROSENBERG, 1976). Assim, o processo de crescimento da organização inovadora (tal como o INI) se beneficia de esforços de inovação (ORGANIZAÇÃO DOS ESTADOS AMERICANOS, 2001).

De acordo com essa nova abordagem da organização, a hipótese comportamental alternativa à da universalidade da inovação poupadora de custos é a de busca de eficiência relativa, responsável pelo interesse no uso de modelos como o Modelo da Análise Envoltória de Dados (Data EnvelopmentAnalysis - DEA) para testar a hipótese de que o desiderato da organização produtiva é a busca de eficiência orientada pela emulação do desempenho dos pares, e não a eficiência absoluta (MANTRI, 2008). Para o objetivo de avaliação, é requerido um tratamento adequado da distinção entre a ineficiência na gestão de fenômenos e variáveis sob controle do administrador e os efeitos de fatores que escapam ao seu controle.

Com vistas a uma medida síntese de avaliação de desempenho, a seleção de um padrão de comparação entre organizações é tratada na literatura sobre avaliação pela identificação da chamada fronteira de eficiência através, por exemplo, dos métodos de cálculo da fronteira não-paramétrica do conjunto das possibilidades de produção empiricamente observadas entre os pares conhecidos da organização estudada. É precisamente o caso do estudo de organizações em que múltiplas atividades responsáveis por ineficiências decorrentes dos problemas de "ajuste mútuo" no curto prazo disputam internamente os recursos disponíveis (MINTZBERG 
et al., 2006). Essas organizações integram uma significativa família de unidades econômicas com tipos de propriedade e atividade diferentes, incluindo as organizações públicas multipropósito de saúde orientadas pelo Modelo de Atenção Integral, como o INI.

\section{Formulação do problema da pesquisa}

Artigo anterior sobre a pesquisa no Lapclin-Chagas (HASSLOCHER-MORENO et al. , 2013) retratou a diversificação da assistência prestada neste laboratório em 2009 - exames de diagnóstico e atenção clínica, o porte alcançado pelas suas atividades de ensino e pesquisa em 2012 e o ganho de eficiência relativa global acumulado no laboratório até 2011.

Tendo em vista a mudança organizacional e a evolução recente da pesquisa clínica em doença de Chagas no INI, o objetivo deste artigo é avaliar o efeito da reestruturação organizacional orientada para a descentralização da tomada de decisão sobre a promoção da pesquisa clínica e o desenvolvimento da atenção integral em doenças infecciosas.

Neste artigo, o problema de avaliação é formulado como um problema de utilização dos novos dados disponíveis a respeito da evolução das quantidades produzidas, das despesas anuais e dos custos unitários efetivos dos laboratórios do INI, com vistas à construção e análise de indicadores que permitam evidenciar os efeitos das novas subestruturas, resultantes da mudança organizacional pró-descentralização no INI, sobre a evolução, no período de análise: da distribuição dos recursos destinados às atividades de atenção clínica entre os seus laboratórios; da produção, assim como do grau de diversificação da assistência e do desempenho em custos da atenção clínica prestada no Lapclin-Chagas; e da eficiência deste laboratório no uso de recursos.

Em suma, o problema da pesquisa é:

(a) testar a hipótese de minimização de custo (promoção da queda do custo unitário efetivo do conjunto das múltiplas atividades da PAI do Lapclin-Chagas entre 2006 e 2011, a preços constantes de 2013);

(b) testar a hipótese de efeito positivo sobre a produção do Lapclin-Chagas no primeiro quinquênio subsequente à reestruturação;

(c) testar a hipótese de efetividade em custo do Lapclin-Chagas (a despesa anual por atividade do laboratório em 2011 é relativamente maior nas atividades com custo unitário menor do que a das outras PAI); e

(d) testar a hipótese de que a reestruturação pró-orientação inovadora proporciona aumento da eficiência técnica relativa do Lapclin-Chagas vis-à-vis às demais PAI do INI no período 2006-2012. 


\section{Método de pesquisa}

O método usado nesta avaliação de eficácia (BANA e COSTA, 2000) é um estudo de caso sobre a nova organização do Lapclin-Chagas, com suporte empírico fornecido, primeiro, pelos indicadores sobre a evolução do volume, da diversificação e das despesas e custos da produção do laboratório, visando avaliar seu crescimento e seu desempenho em custos no período 2006-2011.

Como demarcação da abrangência do Lapclin-Chagas, foram consideradas as atividades de pesquisa, ensino, diagnóstico e atendimento do INI em atenção à coorte de pacientes incluídos nos bancos de dados dos projetos de pesquisa dos seus médicos.

Para assegurar a cooperação do informante, a eliminação da duplicidade de esforços de levantamento foi possível porque as variáveis de volume e diversificação da produção foram quantificadas com dados secundários, compilados nos arquivos de acesso público do INI (JORGE et al., 2006).

A apuração de despesas e custos nas organizações públicas multipropósito de saúde depende da solução de dois problemas enfrentados para aferir estimativas que garantam o incentivo a escolhas de produção genuínas (BIRKINSHAW; HAMEL; MOL, 2008). O primeiro problema é a dificuldade para recensear as despesas de produção (DJELLAL; GALLOUJ, 2005) em nível de microcustos (BREWER; NORREN; GARRISON, 2005), requisito para uma gestão eficiente (MEDICl; MARQUES, 1996). O segundo é a dificuldade para apropriar os custos "indiretos" (NAKAGAWA, 1994) - ou custos "comuns" (MILGROM; ROBERTS, 1992). A apuração das despesas e custos dos laboratórios do INI (WARREN; REEVE; FESS, 2008) recorreu ao método de levantamento de microcustos (HORNGREN; SUNDEM; STRATON, 2004) e ao sistema de Custeio Baseado em Atividades $(A B C)$.

O ABC visa reduzir as distorções provocadas pelo rateio arbitrário dos custos indiretos (NAKAGAWA, 1994) e facilita a análise estratégica dos custos que têm maior impacto no uso de recursos em organizações com elevada participação de custos indiretos e com variado mix de produtos (SOUZA; MAUSS; DIEHL, 2008), tais como as organizações públicas de saúde. Diante do problema de apropriação dos custos por produto, o ABC parte da representação da organização por atividades e processos para então medir relações entre recursos e atividades, denominadas 'direcionadores de custos', a saber, inputs ou atividades que provocam variação no custo total de um objeto de custeio (NAKAGAWA, 1994).

Parte da literatura reconhece o poder do Modelo ABC para explicar as despesas de produção em nível de microcustos que orientam a escolha e a mudança da pauta de produtos competitivos da organização (BIRKINSHAW; HAMEL; MOL, 2008). Como destaca, o método ABC considera, corretamente, que a apropriação dos custos ao preço de venda do produto é orientada para a preservação do incentivo à sua inclusão na pauta de produção da organização. Assim sendo, os procedimentos de apuração dos custos da organização pelo Método $A B C$ são adequados para quantificar as variáveis representativas dos recursos utilizados.

Face à diversidade dos serviços prestados nesses laboratórios, vários itens de despesa em nível de microcustos inscritos no seu orçamento, doravante 
Marcelina Jasé Jarge, Alejandro Marcel Hasslacher Morena, Andréa Silvestre de Sausa, Patrícia Santas Cavalheiro Silva, Georg Michael Lennart Weinberg

denominados Despesas Diretas, concorrem para a formação dos custos unitários efetivos, quais sejam as despesas com: kits ereagentes; serviços de terceiros pessoa física; material de almoxarifado; material de consumo não-almoxarifado; telefonia; amortização de material permanente; manutenção de material permanente; e apoio administrativo interno.

Como prescrito na literatura, para preservar a propriedade de incentivo do método de apuração (ELDENBURG; WOLCOTT, 2007) foram observados os seguintes preceitos: os dados de produção, despesa e material permanente referem-se ao período anual, para expurgar as variações sazonais (MEDICI; MARQUES, 1996); foi considerada a despesa de formação de fundo de reserva para as despesas de capital necessárias à sustentabilidade da organização pública (BRESSER PEREIRA, 1996); a estimativa da "despesa de pessoal" com os produtos produzidos foi calculada como a soma das despesas de remuneração e encargos referentes às parcelas do uso do tempo dos vários especialistas necessários à sua produção (GRÖNROOS, 2003); e a despesa com diagnóstico foi estimada como a despesa relativa ao uso do resultado do exame, lido por interesse de vários projetos e correspondendo a uma parcela da despesa de realização do exame.

Doravante denominadas Despesas Indiretas, foram consideradas as despesas com: pessoal não-remunerado pelo INI; medicamentos de transferência extraorçamentária; apoio administrativo interno; e administração do campus. A elas é destinada boa parte dos recursos do orçamento do "escritório central" da Fiocruz, cujo rateio depende das quantidades de recursos compartilhadas por algumas atividades ou de recursos de uso inespecífico em todas as atividades necessárias a cada produto da pauta de produção do laboratório (JORGE et al., 2006).

Quanto ao efeito da reestruturação sobre a efetividade em custo das atividades de assistência dos laboratórios, esta avaliação considera, primeiro, que as despesas anuais de cada atividade, por laboratório, representam a preferência acerca da distribuição dos recursos do orçamento de cada atividade do INI entre eles. Segundo, considera que as despesas unitárias médias incorridas em cada uma dessas atividades representam a efetividade em custo dos laboratórios no uso dos recursos.

Um diagnóstico global sobre o desempenho em custos da organização visa responder se os recursos de cada atividade são destinados prioritariamente àquelas unidades gestoras de melhor desempenho em custos, contribuindo para a eficiência da organização. No caso do INI, é avaliada a relação entre as variações da despesa anual e do custo unitário de cada laboratório nas suas atividades em 2011, ano de consolidação da inovação organizacional aqui estudado.

O coeficiente de Spearman foi utilizado para identificar o tipo de associação de fato existente entre despesas médias e anuais em cada atividade de assistência dos laboratórios, em 2011 (TRIOLA, 2005). A associação identificada entre os ranks de ordenação das despesas média e anual dos laboratórios, em cada atividade de assistência, permite conhecer a relação empírica entre as variações no valor da despesa anual de cada atividade, por laboratório, e as variações nos valores das respectivas despesas médias (SIEGEL, 1956).

A hipótese correspondente acerca do efeito da reestruturação do INI sobre o desempenho em custos dos laboratórios é de que, na atividade de assistência do laboratório em que a despesa por unidade de produto é maior, a aplicação de 
recursos do orçamento de 2011 é menor, quando comparada com as dotações nela destinadas aos demais laboratórios.

Dada a diversidade de serviços que a PAI do INI abrange, o desempenho do modelo de atenção integral no tratamento da doença de Chagas no Lapclin-Chagas foi em seguida avaliado sob o critério da eficiência no uso dos recursos, a partir da análise dos escores-síntese de eficiência relativa com respeito a uma fronteira de eficiência calculada pelo modelo DEA.

Do total de $14 \mathrm{PAI}$ do INI, foi considerado o desempenho de uma amostra constituída das $8 \mathrm{PAl}$ já estruturadas segundo o Modelo de Atenção Integral à Saúde no período 2002-2012, totalizando 88 unidades de observação: as PAl anuais de doença de Chagas, doenças febris agudas e dengue, vírus T-linfotrópicos humanos, leishmanioses, micoses, toxoplasmose, tuberculose e HIV/aids (JORGE et al., 2006).

Em termos econômicos, a fronteira única plurianual de eficiência calculada para as PAI anuais do INI é a fronteira de produção constituída com a representação das PAl que revelaram as melhores práticas no conjunto dos anos do período analisado, ou seja, a produção máxima empiricamente observada de qualquer PAl entre a população estudada, obtida a partir de sua dotação efetiva de recursos. No caso específico da aplicação da DEA neste artigo, a comparação da eficiência de uma dada Unidade Tomadora de Decisão (DMU) se dá pela revelação do desempenho das outras DMU sob análise. O objetivo é obter uma superfície envoltória - fronteira de eficiência - formada pelas DMU com melhor desempenho.

Aplicado a um conjunto de $\mathrm{K}$ organizações que utilizam $\mathrm{N}$ insumos para produzir $\mathrm{M}$ produtos, o Modelo DEA permite calcular os escores-síntese $E_{i}$ de cada organização:

$E_{i}=Y_{i} / X_{i}=\left(A_{1 i} \cdot O_{1 i}+\ldots+A_{M i} \cdot O_{1 i} I_{M i}\right) /\left(B_{1 i} \cdot I_{1 i}+\ldots+B_{N i} \cdot I_{N i}\right)(1)$

em que os $O$ representam produtos; os I representam insumos; e os $A_{i j}$ e $B_{i j}$ representam pesos, ou seja, a importância relativa de cada produto e insumo. Do ponto de vista matemático, esse modelo presta-se a identificar os pesos que maximizam o escore atribuível à avaliação da unidade econômica pela análise de eficiência. Assim, é possível hierarquizar as organizações segundo sua eficiência técnica relativa, com a especificação de um padrão de comparação.

No modelo gráfico da FIG 1, por exemplo, face à situação relativa de $\cup_{i}$, quando comparado o seu desempenho com o de $U_{1}$ e $U_{2}$, conclui-se que, entre essas três $D M U, U_{i}$ é a que pertence à fronteira eficiente, já que $\cup_{1}$ utiliza o mesmo montante de recursos $X_{i}$ para produzir $Y_{1}$. Ou seja, menos do que $Y_{i}$, enquanto $U_{2}$ produz 0 mesmo que $U_{i}$, mas utiliza um volume de recursos $X_{2}$ maior do que $X_{i}$. Nesse sentido, a ineficiência técnica de $U_{1}$ e $U_{2}$ é expressa pelos escores-síntese menores que o de $U_{i}$.

Convenciona-se que, para todas as $D M U$ situadas na fronteira, $E_{L}=Y_{L} / X_{L}=1$, ou seja, que todas elas se caracterizam por uma situação de eficiência plena: DMU $100 \%$ eficiente. Assim sendo, a eficiência relativa $E_{1}$ de uma $D M U U_{1}$, cujo ponto representativo não faz parte da fronteira eficiente, pode ser medida como $Y_{1} / X_{l}<1$, e este é um escore-síntese para o seu desempenho. 


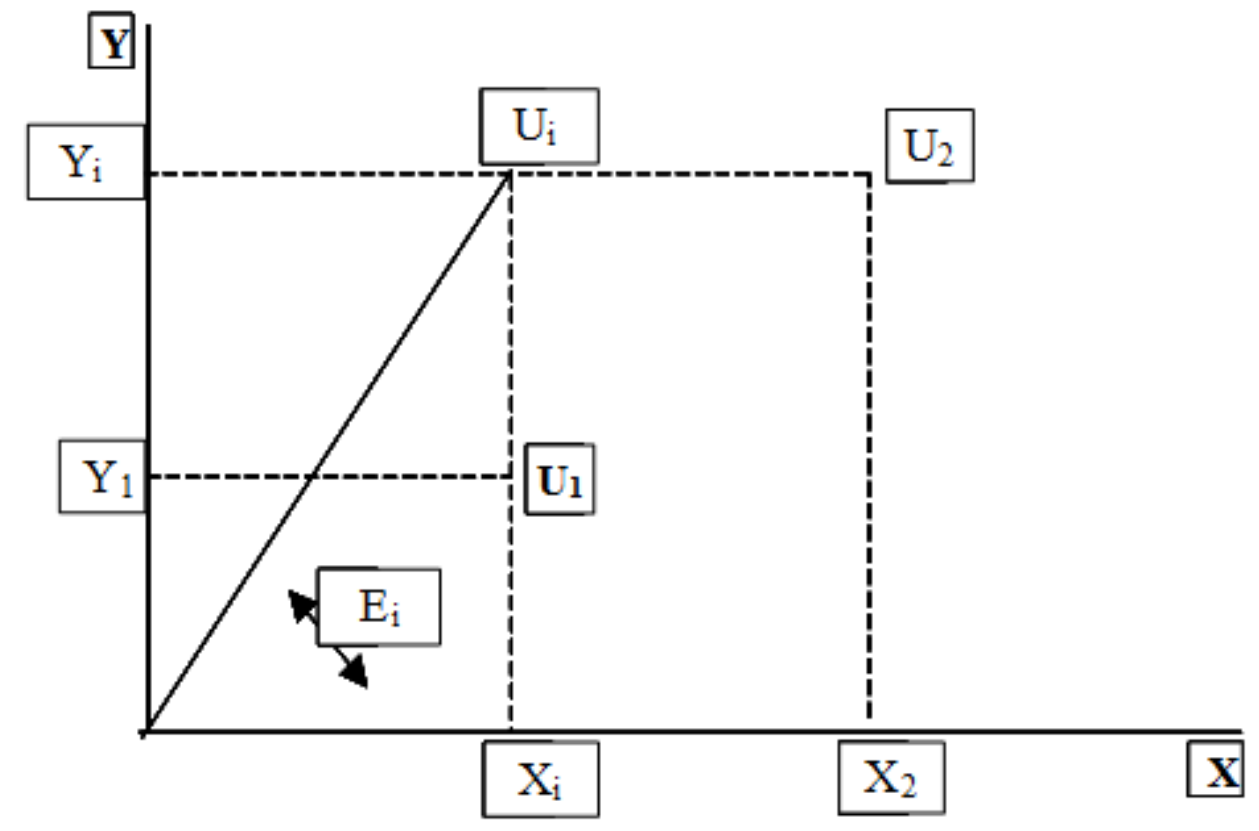

Figura 1 - Representação gráfica do escore-síntese de Eficiência Técnica Fonte: Elaboração dos autores.

O modelo DEA foi escolhido para a análise de eficiência das PAI porque: (a) distingue a ineficiência na gestão dos fenômenos sob o controle do gerente vis-à-vis os efeitos dos outros fatores fora do controle do administrador; (b) sua utilização incentiva o agente da produção a informar da "melhor maneira", tornando a avaliação imune ao blefe; (c) não requer prévio conhecimento da tecnologia para representar a organização avaliada; (d) focaliza observações individuais, ao invés de médias da população; (e) calcula uma medida agregada individual da DMU, quanto à utilização de insumos para produzir os produtos almejados; (f) usa múltiplos produtos e insumos simultaneamente, possíveis de serem considerados em diferentes unidades de medida; e (g) satisfaz o critério de equidade na avaliação relativa de cada DMU.

Entre os modelos DEA para análise, foi escolhido o Modelo DEA com Retornos Variáveis de Escala Orientado para o Produto (DEA-BCC-O), que possibilita analisar processos produtivos de DMU com portes distintos, tais como as PAI. O modelo DEA-BCC-O é utilizado para calcular a produção máxima da organização em análise, quando mantido constante o consumo agregado de insumos, sendo considerado mais pertinente ao objetivo do presente artigo, uma vez que o orçamento do INI e das PAI é estabelecido a priori e o alvo do gerente local é, antes, o de aumentar os resultados obtidos do que reduzir os insumos utilizados.

Finalmente, as variáveis de insumo do modelo calculado foram selecionadas por sua importância na despesa: (a) horas-médico - tempo dedicado por profissionais médicos a cada PAl -; e (b) custeio exceto pessoal - despesa anual com medicamentos, reagentes para exames e material hospitalar por PAI. Os produtos foram eleitos por sua importância para o resultado das PAI: (a) exames - quantidade de exames realizados distribuída por PAI -; (b) consultas - quantidade de consultas prestadas por PAI -; (c) internações - quantidade de dias de internação por PAI -; (d) produção científica - quantidade de artigos publicados por PAI -; (e) coorte - 
quantidade de novos pacientes de pesquisa por PAI -; e (f) ensino - quantidade de dissertações e teses defendidas por PAI.

\section{Análise dos resultados}

As medidas da associação entre as variáveis representativas da participação dos vários laboratórios na despesa anual de cada atividade de assistência e do seu desempenho em custos nesta atividade, obtidas com o cálculo do coeficiente de Spearman para o teste da correlação dos ranks destas variáveis em 2011, são esclarecedoras sob a ótica da análise de efetividade em custos: os valores negativos das correlações na TAB 1 indicam associação inversa entre as variáveis de despesa anual e média e efetividade em custos na atividade - os recursos usados nos laboratórios com melhor desempenho em custos são maiores. Vale notar, porém, que apenas dois coeficientes são significativos a 5\% ou menos (ZAR, 1972).

Tabela 1 - INI - Efetividade em Custo da Distribuição da Despesa da Atividade de Assistência por Laboratório: 2011

\begin{tabular}{|c|c|c|c|c|c|}
\hline & \multirow{2}{*}{$R, R E$} & \multicolumn{4}{|c|}{ DD-ANO: 2011} \\
\hline & & Ambulatório & Hospital-Dia & Internação & CTI \\
\hline \multirow{4}{*}{ 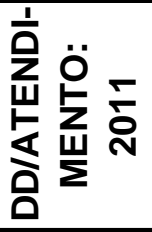 } & Ambulatório & $-0,72(\mathrm{R})^{\star \star}$ & - & - & \\
\hline & Hospital-Dia & - & $-0,56$ (RE) & - & \\
\hline & Internação & - & - & $-0,69(R)^{\star \star \star}$ & \\
\hline & CTI & & & & $-0,73(\mathrm{RE})^{\star *}$ \\
\hline
\end{tabular}

Fonte: INI.

$\mathrm{R}, \mathrm{RE}$ - Coeficiente de Correlação de Spearman, respectivamente sem e com empate de postos.

** : $p<5 \%$

$\star \star \star *: p<10 \%$

Como a TAB 2 permite visualizar, o número anual de projetos de pesquisa clínica em desenvolvimento no Lapclin-Chagas quadruplicou, seguido do crescimento do número de consultas médicas-ano de $9 \%$, dos procedimentos de enfermagem prestados no ano de $140 \%$ e dos dias-ano de internação de $105 \%$ no período 2006 2011, todos indicativos do efeito positivo sobre a produção do laboratório no primeiro quinquênio subsequente à sua reestruturação. 
71 Marcelina Jasé Jarge, Alejandra Marcel Hasslacher Morena, Andréa Silvestre de Sousa, Patrícia Santas Cavalheira Silva, Georg Michael Lennart Weinberg

Tabela 2 - Lapclin- Chagas - Evolução da Produção Anual por Atividade: 2006-2011

\begin{tabular}{lcc}
\hline \multirow{2}{*}{ Atividade } & \multicolumn{2}{c}{ Quantidade-ano } \\
\cline { 2 - 3 } & $\mathbf{2 0 0 6}$ & $\mathbf{2 0 1 1}$ \\
\hline Ambulatório & 3.019 & 3.100 \\
Consulta de Infectologista & 1.760 & 2.122 \\
Consulta de Médico Não-Infectologista & 7.140 & 767 \\
Consulta de Outras Especialidades & 2.701 & 6.476 \\
Procedimentos de Enfermagem $^{\text {Internação }}$ & 268 & 548 \\
Hospital-dia $^{(1)}$ & 1 & 10 \\
Ensino $^{(1)}$ & 18 & 29 \\
Pesquisa $^{(2)}$ & 4 & 18 \\
\hline
\end{tabular}

Fonte: INI.

Notas:

(1) Egressos de cursos.

(2) Projetos de pesquisa em desenvolvimento.

Os

Projetos de pesquisa clínica em desenvolvimento no Lapclin-Chagas: Estudo Longitudinal da Morbimortalidade Cardíaca na Fase Crônica da Doença de Chagas; Estudo Clínico-Epidemiológico da Fase Crônica da Doença de Chagas; Testes Diagnósticos para o Rastreamento de Doença de Chagas; e Tratamento Etiológico/Específico da Doença de Chagas (HASSLOCHER-MORENO et al., 2013). Com respeito à atividade de ensino do Lapclin-Chagas nas disciplinas de pósgraduação stricto sensu das áreas de cardiologia (UFRJ) e de doenças infecciosas (INI), foram apresentadas 7 teses de doutorado e 28 dissertações de mestrado até 2012 (HASSLOCHER-MORENO et al., 2013).

Na TAB 3, é apresentada a evolução, no quinquênio analisado, do volume por tipo de serviço prestado e da diversificação por tipos dos exames e procedimentos clínicos realizados em atenção à coorte de cerca de 2200 pacientes do LapclinChagas, bem como das despesas anuais e dos custos unitários efetivos com recursos do orçamento do INI (Despesa Direta) e do orçamento de gastos do "escritório central" da Fiocruz (Despesa Indireta). No período, a quantidade anual de exames e procedimentos realizados em atenção à coorte de pacientes do laboratório cresceu de 34.935 para 47.478 (36\%) e o número de tipos de 236 para 265 (12\%). 
Tabela 3 - Lapclin- Chagas - Atividades de Atendimento, Diagnóstico e Suporte: 2006-2011

\begin{tabular}{|c|c|c|c|c|c|c|c|c|}
\hline \multirow[b]{2}{*}{ DESCRIÇÃO } & \multicolumn{4}{|c|}{2006} & \multicolumn{4}{|c|}{2011} \\
\hline & QTD. & TIPOS & $\begin{array}{c}\text { DDI-ANO } \\
\left({ }^{*}\right)\left({ }^{* *}\right)\end{array}$ & $\begin{array}{c}\text { DDII } \\
\text { ATENDI- } \\
\text { MENTO } \\
(*)\end{array}$ & QTD. & TIPOS & $\begin{array}{c}\text { DDI-ANO } \\
\left(^{*}\right)\left({ }^{* *}\right)\end{array}$ & $\begin{array}{c}\text { DDII } \\
\text { ATENDI- } \\
\text { MENTO } \\
(*)\end{array}$ \\
\hline Consultas de Infectologista & 3.019 & 2 & $581.860,43$ & 192,73 & 3.100 & 2 & $254.575,38$ & 82,12 \\
\hline Consultas de Médico Não-Infectologist & 1.770 & 2 & $164.695,03$ & 93,03 & 2.122 & 2 & $241.250,36$ & 113,69 \\
\hline Consultas de Outras Especialidades & 4.140 & 2 & $473.012,85$ & 114,25 & 767 & 2 & $298.327,94$ & 389,00 \\
\hline Procedimentos de Ambulatório & 117 & 14 & $27.241,32$ & 232,10 & 145 & 16 & $61.007,55$ & 420,06 \\
\hline Procedimentos de Enfermagem & 2.701 & 45 & $95.593,25$ & 35,39 & 6.476 & 81 & $112.499,59$ & 17,37 \\
\hline Atendimentos de Hospital-Dia & 1 & - & $6.708,32$ & $6.708,32$ & 10 & - & $15.284,79$ & $1.528,48$ \\
\hline \multicolumn{9}{|l|}{ Leito-Dia (com Refeição e } \\
\hline Medicamento) & 268 & - & $426.008,15$ & $1.589,58$ & 548 & - & $957.522,03$ & $1.747,30$ \\
\hline Leitos-Dia & 268 & - & $341.766,20$ & $1.275,25$ & 548 & - & $856.768,56$ & $1.563,45$ \\
\hline Paciente de Leito-Dia & 25 & - & $341.766,20$ & $13.670,65$ & 46 & - & $856.768,56$ & $18.625,40$ \\
\hline \multicolumn{9}{|l|}{ Leito-Dia - CTI (com Refeição e } \\
\hline Medicamento) $(* \star \star)$ & - & - & - & - & 221 & - & $332.211,65$ & $1.503,22$ \\
\hline Leitos-Dia - CTI (***) & - & - & - & - & 221 & - & $283.338,66$ & $1.282,08$ \\
\hline Paciente de Leito-Dia - CTI (***) & - & - & - & - & 19 & - & $283.338,66$ & $14.912,56$ \\
\hline Lab. Anatomia Patológica & 462 & 4 & $118.009,95$ & 255,45 & 910 & 4 & $154.617,94$ & 169,93 \\
\hline Lab. Bacteriologia e Bioensaios & 1.315 & 19 & $122.734,40$ & 93,32 & 2.087 & 18 & $172.446,68$ & 82,64 \\
\hline Lab. Imagem & 1.131 & 60 & $291.959,03$ & 258,03 & 611 & 57 & $314.900,01$ & 515,54 \\
\hline Lab. Imunodiagnóstico & 2.806 & 21 & $150.569,13$ & 53,66 & 2.786 & 21 & $137.439,54$ & 49,34 \\
\hline Lab. Micologia & 13 & 2 & $1.525,80$ & 116,91 & 4 & 3 & 832,77 & 188,92 \\
\hline Lab. Parasitologia & 749 & 8 & $77.558,28$ & 103,51 & 485 & 7 & $60.106,66$ & 124,04 \\
\hline Lab. Análises Clínicas & 16.148 & 57 & $487.038,26$ & 30,16 & 26.373 & 52 & $461.430,32$ & 17,50 \\
\hline Dispensação de Ampola & 5.300 & - & $2.516,35$ & - & 100 & - & 60,54 & - \\
\hline Dispensação de Cápsula & 10.000 & - & 316,88 & - & 2.000 & - & 89,69 & - \\
\hline Dispensação de Comprimido & 411.000 & - & $318.230,10$ & - & 265.020 & - & $130.188,25$ & - \\
\hline Refeições no Hospital-Dia & 1 & - & 6,93 & - & 10 & - & 296,51 & - \\
\hline Refeições no Ambulatório & N. D. & - & $28.908,21$ & - & N. D. & - & $30.679,35$ & - \\
\hline Refeições no Internação & 268 & - & $57.553,30$ & - & 548 & - & $93.517,75$ & - \\
\hline Refeições no CTI (***) & 0 & - & 0,00 & - & 221 & - & $37.714,27$ & - \\
\hline Apoio Administrativo Interno & - & - & $376.419,80$ & - & - & - & $503.297,12$ & - \\
\hline Apoio Administrativo do Campus & - & - & $368.390,41$ & - & - & - & $537.922,70$ & - \\
\hline
\end{tabular}

Finalmente, o cálculo das despesas com recursos inscritos no orçamento do INI (DD) pode fornecer um diagnóstico agregado, sob a ótica da minimização de custos, sobre a evolução do desempenho em custos do conjunto dos serviços em cada atividade de assistência do Lapclin-Chagas entre 2006 e 2011. Como se pode verificar na TAB 4, os custos unitários efetivos das consultas médicas, dos procedimentos de enfermagem, do dia de internação e do atendimento de hospitaldia caíram. 
Marcelina Jasé Jarge, Alejandro Marcel Hasslacher Moreno, Andréa Silvestre de Sausa, Patrícia Santas Cavalheira Silva, Gearg Michael Lennart Weinberg

Tabela 4 - Lapclin-Chagas - Despesa Anual e Custo Unitário por Atividade de Assistência: 2006-2011

\begin{tabular}{|c|c|c|c|c|c|}
\hline \multirow{2}{*}{ ATIVIDADE } & \multicolumn{2}{|c|}{ DD-ANO $\left(^{*}\right)\left({ }^{* *}\right)$} & \multicolumn{3}{|c|}{ DD/UNIDADE-SERVIÇO (*) } \\
\hline & 2006 & 2011 & 2006 & 2011 & 2006-2011 \\
\hline AMBULATÓRIO & $300.331,33$ & $241.307,34$ & n.a. & n.a. & n.a. \\
\hline Consulta de Infectologista & $64.480,23$ & $64.375,83$ & 21,36 & 20,77 & DIMINUIU \\
\hline Consulta de Médico Não-Infectologista & $48.599,52$ & $39.779,50$ & 27,45 & 18,75 & DIMINUIU \\
\hline Consulta de Outras Especialidades & $112.470,59$ & $32.963,55$ & 27,17 & 42,98 & AUMENTOU \\
\hline Procedimentos Médicos de Ambulatório & 15,75 & $16.329,03$ & 0,13 & 112,36 & AUMENTOU \\
\hline Procedimentos de Enfermagem & $74.765,24$ & $87.859,42$ & 27,68 & 13,57 & DIMINUIU \\
\hline INTERNAÇÃO & $238.899,35$ & $425.283,50$ & 891,42 & 776,06 & DIMINUIU \\
\hline INTERNAÇÃO - CTI & $-\quad(* \star \star)$ & $203.562,48$ & - & 921,10 & - \\
\hline HOSPITAL-DIA & $5.506,19$ & $8.659,02$ & $5.506,19$ & 865,90 & DIMINUIU \\
\hline
\end{tabular}

$\left(^{\star}\right)$ Em R\$ a preços de 2013.

(**) DD - Despesa Direta (inscrita no orçamento do IPEC).

$\left({ }^{\star \star \star}\right)$ CTI instalado em 2007.

Quantificadas as oito variáveis de insumos e de produtos das PAI do INI e calculado o modelo DEA-BCC-O pela solução do Problema de Programação Linear subjacente através do software FrontierAnalyst ${ }^{\circledR}$, obtêm-se, então, os escores de eficiência técnica relativa de cada PAI anual para o período 2002-2012 apresentados na TAB 5 e na FIG 2.

Tabela 5- Escores-síntese Anuais de Eficiência Técnica Relativa das PAI -2002-2012

\begin{tabular}{llllllllllll}
\hline $\begin{array}{l}\text { Ano } \\
\text { PAI }\end{array}$ & $\mathbf{2 0 0 2}$ & $\mathbf{2 0 0 3}$ & $\mathbf{2 0 0 4}$ & $\mathbf{2 0 0 5}$ & $\mathbf{2 0 0 6}$ & $\mathbf{2 0 0 7}$ & $\mathbf{2 0 0 8}$ & $\mathbf{2 0 0 9}$ & $\mathbf{2 0 1 0}$ & $\mathbf{2 0 1 1}$ & $\mathbf{2 0 1 2}$ \\
\hline Chagas & 0,79 & 0,81 & 0,73 & 0,86 & 0,76 & 0,77 & 0,72 & 0,73 & 0,54 & 0,88 & 0,87 \\
DFA/Dengue & 0,88 & 1,00 & 0,96 & 0,99 & 0,99 & 1,00 & 1,00 & 1,00 & 0,79 & 1,00 & 1,00 \\
HTLV & 1,00 & 0,85 & 0,98 & 0,73 & 0,72 & 0,87 & 0,83 & 0,81 & 0,52 & 0,88 & 0,87 \\
Leishmaniose & 1,00 & 1,00 & 1,00 & 0,91 & 0,93 & 0,88 & 0,74 & 0,81 & 0,95 & 1,00 & 1,00 \\
Micoses & 1,00 & 1,00 & 1,00 & 1,00 & 0,84 & 1,00 & 0,86 & 1,00 & 1,00 & 1,00 & 1,00 \\
Toxoplasmose & 1,00 & 1,00 & 1,00 & 1,00 & 0,87 & 0,91 & 0,86 & 0,92 & 0,60 & 0,92 & 0,96 \\
Tuberculose & 1,00 & 1,00 & 1,00 & 1,00 & 0,89 & 0,95 & 0,86 & 0,84 & 0,60 & 0,89 & 0,95 \\
HIV & 1,00 & 1,00 & 0,85 & 1,00 & 0,91 & 1,00 & 0,96 & 1,00 & 1,00 & 1,00 & 1,00 \\
\hline Média & 0,96 & 0,96 & 0,94 & 0,93 & 0,86 & 0,92 & 0,85 & 0,89 & 0,75 & 0,95 & 0,96 \\
\hline
\end{tabular}

Fonte: Elaboração dos autores.

Variáveis de Input: Hora-Médico, Outros Custeios.

Variáveis de Output: Exame, Consulta, Internação, Produção Científica, Coorte, Dissertações e Teses.

O uso de testes não paramétricos de aferição da precisão estatística dos resultados da Análise de Eficiência desenvolvida com o modelo adotado permitiu confirmar que as variações dos escores-síntese anuais de eficiência técnica relativa obtidos são estatisticamente significativas - veja Jorge et al. (2006). 


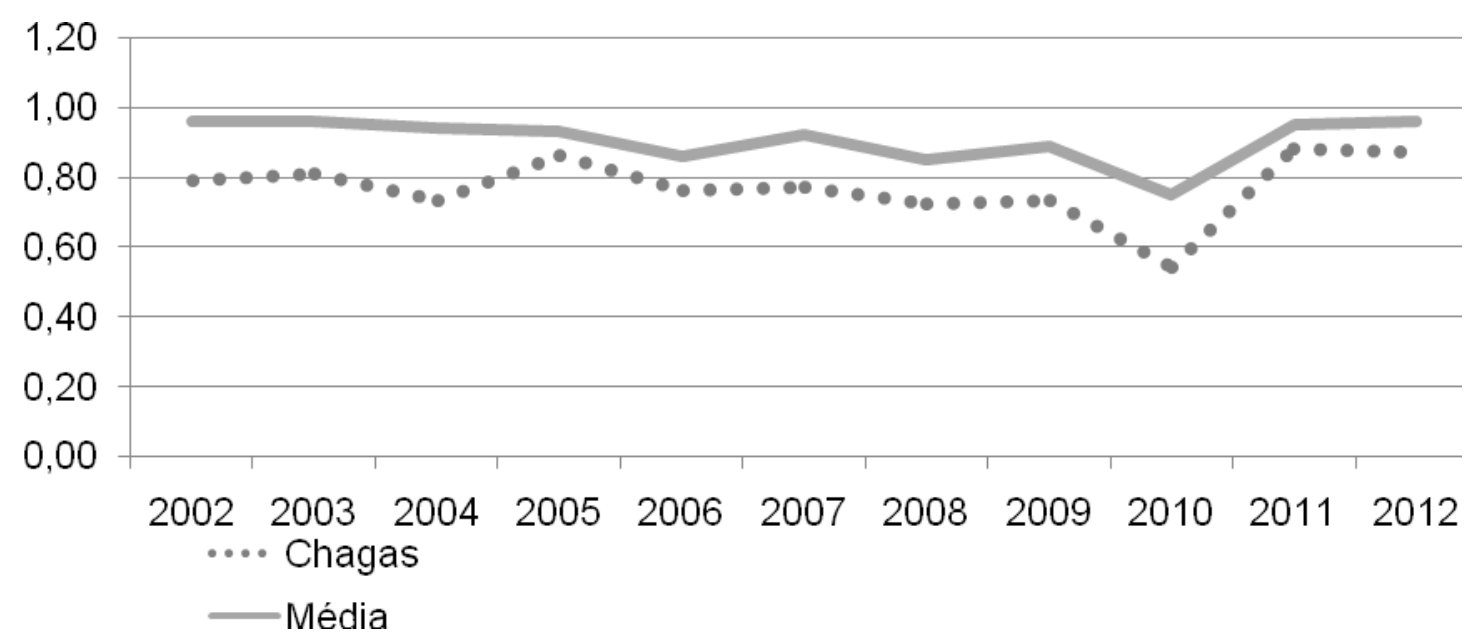

Figura 2- Evolução Anual da Eficiência Técnica Relativa das PAI - 2002-2012 Fonte:Elaboração dos autores.

\section{Conclusões}

A pesquisa clínica, como atividade de produção de conhecimento, é uma atividade tipicamente desenvolvida nas organizações públicas de saúde, e a pesquisa clínica sobre doenças infecciosas, nas organizações públicas multipropósito de saúde; o seu desenvolvimento implica relações de intercâmbio de conhecimento mais efetivas entre as atividades de pesquisa e de assistência.

A reconstrução da trajetória da pesquisa clínica na Fiocruz à luz do referencial conceitual proposto na Teoria das Configurações de Mintzberg (1995) é esclarecedora sobre o acerto na escolha da abordagem de estudo de caso único sobre a eficácia do modelo (BANA e COSTA, 2000) do Lapclin-Chagas para esta avaliação.

No caso particular dos laboratórios do INI, a expectativa com respeito à reestruturação do Lapclin-Chagas em 2007, por exemplo, foi de lograr a expansão das suas atividades de pesquisa, ensino e assistência, bem como a diversificação e a efetividade em custos dos procedimentos de atenção integral à coorte dos seus pacientes.

A avaliação evidenciou o elevado grau de diversificação dos procedimentos do Lapclin-Chagas no período da análise: os pacientes da coorte do Lapclin-Chagas recebem atenção de caráter interdisciplinar e multiprofissional, e o modelo de tratamento da doença de Chagas do Lapclin-Chagas é orientado para a promoção da saúde, o suporte em evidências e o atendimento humanizado, com envolvimento familiar e social na atenção, assistência de proteção social e controle do comportamento de risco.

Por sua vez, a avaliação do desempenho em custos da assistência do LapclinChagas com o método $A B C$ revelou evolução heterogênea por procedimento.

O resultado da análise de eficácia da estrutura do INI assim concebida permite concluir que o crescimento da atividade de pesquisa do Lapclin-Chagas, a partir da 
Marcelina Jasé Jarge, Alejandro Marcel Hasslacher Morena, Andréa Silvestre de Sausa, Patrícia Santas Cavalheiro Silva,

inovação organizacional na sua estruturação, foi compatível com o crescimento e a diversificação da assistência e, sob a ótica do desempenho em custos do conjunto dos laboratórios, permite confirmar a hipótese de associação entre a reestruturação e a efetividade em custos da distribuição, entre os laboratórios, dos recursos orçamentários do INI para cada atividade de assistência. Em outras palavras, fortaleceu os laboratórios mais eficientes.

Não foi possível, no entanto, confirmar a hipótese de comportamento poupador de custos no sentido estrito da racionalidade microeconômica: a preços de 2013, a expansão do Lapclin-Chagas não ocorreu com redução dos custos unitários dos procedimentos de consulta de ambulatório de especialidades não-médicas, nem dos procedimentos médicos de ambulatório no período 2006-2011.

A seguir, a Análise de Eficiência mostrou que a eficiência técnica relativa das PAI cresceu ao longo do tempo, permitindo confirmar a hipótese de associação positiva entre a distribuição da despesa anual do INI por PAI e a busca das melhores práticas na gestão destes recursos nas PAI.

Como contribuição ao conhecimento, o artigo associou a dinâmica de mudança da estrutura organizacional do INI no período 2002-2012 e a orientação pró-eficiência da estratégia de produção multipropósito da pesquisa clínica em doenças infecciosas adotada em 1999 à hipótese de busca de eficiência orientada pelo desempenho dos pares adotada pela Análise de Eficiência (MANTRI, 2008).

Como contribuição gerencial, o artigo confirmou a associação entre a nova estrutura de organização da assistência associada à pesquisa clínica no INI e a motivação pró-eficiência relativa, exibindo evidências sobre o benefício potencial da sua replicação para a expansão e o aperfeiçoamento do tratamento da doença de Chagas.

Nesse sentido, demonstrou-se o poder explicativo do modelo DEA para o conhecimento das organizações multipropósito, embora a análise de um caso único imponha limitações à generalização dos resultados obtidos. A DMU analisada é a PAI, o que constitui uma limitação à possibilidade de generalização dos resultados obtidos sobre a motivação pró-eficiência relativa das estruturas organizadas, segundo o Modelo de Atenção Integral. Essa estrutura, porque realiza atividades de pesquisa, difere das organizações de tipos General Hospital e Academic Medical Center, avaliadas em outros estudos orientados para a investigação do conjunto do sistema de saúde (OZCAN, 2008).

Do ponto de vista do estudo da busca de eficiência na organização pública multipropósito de saúde, porém, a estrutura gerencial interna de solução dos problemas de coordenação e compromisso é importante para concretizar o objetivo de expansão. Portanto, as metas relacionadas com a promoção da expansão através da diversificação da pauta de produção definem a DMU relevante para a avaliação e as variáveis de insumo e produto do modelo DEA (JORGE et al., 2006; SHERMAN; ZHU, 2006). 


\section{Referências}

KAPLAN, R. S., ATKINSON, A. A., YOUNG, S., \& BANKER, R. Contabilidade gerencial. São Paulo: Atlas. 2000

BANA e COSTA, C. A. Entrevista. Informe n. 20. Boletim da SOBRAPO, Rio de Janeiro, 2000.

BIRKINSHAW, J.; HAMEL, G.; MOL, M. J. Management innovation. Academy of Management Review, Nova York, v. 33, n. 4, p. 825-845, 2008.

BRESSER PEREIRA, L. C. Crise econômica e reforma do estado no Brasil: para uma nova interpretação da América Latina. São Paulo: Editora 34, 1996.

BREWER, P. C.; NOREEN, E. W.; GARRISON, R. H. Managerial accounting. New York: McGraw-Hill, 2005.

CUNILL GRAU, N.; OSPINA BOZZI, S. "La evaluación de los resultados de la gestión pública: una herramienta técnica y política". In: CLAD/AECI, CUNILL GRAU, N.; OSPINA BOZZI, S. (Eds.), Evaluación de Resultados para una Gestión Pública Moderna y Democrática: experiencias latinoamericanas, Caracas, 2003.

DJELLAL, F.; GALLOUJ, F. Mapping innovation dynamics in hospitals. Research Policy, v. 34, n. 6, p. 817-835, 2005.

ELDENBURG, L. G.; WOLCOTT, S. K. Gestão de Custos: como medir, monitorar e motivar o desempenho. Rio de Janeiro: LTC, 2007.

FIOCRUZ, V Congresso Interno - Relatório Final da Plenária Extraordinária. Diretrizes para a Adequação da Estrutura Organizacional da Fiocruz, 2006. Disponível em: $<$ http://www.fiocruz.br/congressointerno/media/relatorio_final_vci_plenaria.pdf>. Acesso em: 15 jul. 2013.

GRÖNROOS, C. Marketing: gerenciamento e serviços. Rio de Janeiro: Elsevier, 2003.

HARRIS, J. E. The internal organization of hospital: some economic implications. The Bell Journal of Economics, Santa Monica/CA, v. 8, n. 2, p. 467-482, 1977.

HASSLOChER-MORENO, A. M., JORGE, M. J., Sousa, A. S. D., BRASIL, P. E. A. A. D., XAVIER, S. S., BARRETO, N. D. B., ... \& AVELLAR, A. M. Atenção integral e eficiência no Laboratório de Pesquisa Clínica em Doenças de Chagas do Instituto de Pesquisa Clínica Evandro Chagas, 2009-2011. Epidemiologia e Serviços de Saúde, v.22, n.2, p. 295-306. 2013

HORNGREN, C. T.; SUNDEM, G. L.; STRATON, W. O. Contabilidade gerencial.12. ed. São Paulo: Pearson Prentice Hall, 2004.

IVERS, L. C., APPLETON, S. C., WANG, B., JEROME, J. G., CULLEN, K. A., \& FAWZI, M. C. S. HIV-free survival and morbidity among formula-fed infants in a prevention of mother-tochild transmission of HIV program in rural Haiti. AIDS research and therapy, v.8, n.1, p. 37. 2011. 
Marcelina Jasé Jarge, Alejandra Marcel Hasslacher Moreno, Andréa Silvestre de Sousa, Patrícia Santas Cavalheira Silva, Georg Michael Lennart Weinberg

JOGARATNAM, G.; CHING-YICK TSE, E. Entrepreneurial orientation and the structuring of organizations. International Journal of Contemporary Hospitality Management, v. 18, n. 6, p. 454-468, 2006.

JORGE, M. J., AVELLAR, C. M., FERREIRA, D. S., BATISTA, D. L., KWASINSKI, E., \& BUZANOVSKY, N. Efetividade em custo e análise de eficiência de programas em organizações complexas a experiência do IPEC/FIOCRUZ. Ciudad de México: FUNSALUD. 2006

KNAUL, F. M., GONZÁLEZ-PIER, E., GÓMEZ-DANTÉS, O., GARCÍA-JUNCO, D., ARREOLA-ORNELAS, H., BARRAZA-LLORÉNS, M., ... \& KERSHENOBICH, D. The quest for universal health coverage: achieving social protection for all in Mexico. The Lancet, v.380, n. 9849, p.1259-1279. Out. 2012.

LA FORGIA, G. M.; COUTTOLENC, B. F. Hospital performance in Brazil: the search for excellence. Washington, D.C.: The World Bank, 2008.

LEIBENSTEIN, H. Allocative efficiency vs. "X" - efficiency. American Economic Review, Pittsburgh/PA, v. 56, p. 392-415, 1966.

MANTRI, J. K. (Ed.) Research methodology on data envelopment analysis (DEA). Boca Raton, FL: Universal Publishers, 2008.

MEDICI, A. C.; MARQUES, R. M. Sistemas de custos como instrumento de eficiência e qualidade dos serviços de saúde. Cadernos FUNDAP, São Paulo, v. 19, p. 47-59, jan./abr. 1996.

MILGROM, P.; ROBERTS, J. Economics, organization and management. New York: Prentice Hall, 1992.

MINTZBERG, H.. O Processo da Estratégia-4. Bookman Editora. 2006

MINTZBERG, H. Criando organizações eficazes. São Paulo: Atlas, 1995.

MORTON, N. A.; HU, Q. Implications of the fit between organizational structure and ERP: a structural contingency theory perspective. International Journal of Information Management, v. 28, p. 391-402, 2008.

NAKAGAWA, M. ABC: Custeio baseado em atividades. São Paulo: Atlas, 1994.

OLIVEIRA, D. K. S.; NASCIMENTO, D. D. G.; MARCOLINO, F. F. Perceptions of Family Caregivers and Professionals in the Family Health Strategy Related to the Care and Neuropsychomotor Development of Children. Journal of Human Growth and Development, v. 22, n. 2, p. 142-150, 2012.

ORGANIZAÇÃO DOS ESTADOS AMERICANOS. Normalización de indicadores de innovación tecnológica en América Latina y el Caribe: manual de Bogotá. Bogotá: OEA, 2001.

OZCAN, Y. A. Health care bench marking and performance evaluation: an assessment using data envelopment analysis (DEA). Virginia: Springer, 2008.

ROBINSON, R. B. Creating a 21st century entrepreneurial organization. Academy of Entrepreneurship Journal, v. 8, n. 1. p. 321-332, 2007.

REUNA, Belo Horizonte - MG, Brasil, v.21, n.4, p.57-80, Dut. - Dez. 20I6 - ISSN 2179-8834 
ROMER, P. M. The origins of endogenous growth. Journal of Economic Perspectives, Pitsburgo, v. 8, n.1, p. 3-22, 1994.

ROSENBERG, N. Perspectives on technology. Cambridge: Cambridge University Press, 1976.

ROSS, C. L. Integral Healthcare: The Benefits and Challenges of Integrating Complementary and Alternative Medicine with a Conventional Healthcare Practice. Integrative Medicine Insights, v. 4, p. 13-20, 2009.

SHERMAN, D. H.; ZHU, J. Service productivity management: improving service performance using data envelopment analysis (DEA). Boston, MA: Springer, 2006.

SIEGEL, S. Nonparametric statistics for the behavioral sciences. New York: McGraw-Hill, 1956.

SOUZA, M. A. de; MAUSS, C. V.; DIEHL, C. A. A gestão pública por resultados e a avaliação de desempenho. In: Congresso USP Controladoria e Contabilidade. São Paulo: USP, 2008.

TRIOLA, M. F. Introdução à estatística. 9. ed. Rio de Janeiro: LTC, 2005.

ULLOA GONZALEZ, A.; BARRANTES MONGE, M. C. Bioética em Nicaragua. Acta Bioethica, Santiago, v. 14, n. 2, p. 200-205, 2008.

VARIAN, H. R. Microeconomia: princípios básicos. Rio de Janeiro: Campus, 2006.

WARREN, C. S.; REEVE, J. M.; FESS, P. E. Contabilidade gerencial. 2. ed. São Paulo: Thomson Pioneira, 2008.

ZAR, J. H. Significance testing of the Spearman Rank Correlation Coefficient. Journal of the American Statistical Association, v. 67, n. 339, p. 578-80, 1972. 
79 Marcelina Jasé Jarge, Alejandra Marcel Hasslacher Morena, Andréa Silvestre de Sousa, Patrícia Santas Cavalheira Silva, Gearg Michael Lennart Weinberg 